\title{
Inventory of cyanobacteria and microalgae cited for the National Park of the Chapada dos Guimarães (MT) and 80 new records from the region
}

\author{
Gisele Fernanda Pereira Assis ${ }^{I *}$ (), Samiris Pereira da Silva ${ }^{I}$, Ludmylla Fernanda de Siqueira Silva ${ }^{\text {, }}$ \\ Regiane Luiza da Costa ${ }^{1}$, Luany Weiler da Fonseca ${ }^{2}$,Ermelinda Maria De-Lamonica-Freire ${ }^{2}$, \\ Daniela Maimoni de Figueiredo ${ }^{3}$ \& Márcia Teixeira de Oliveira ${ }^{1}$ \\ ${ }^{1}$ Universidade Federal de Mato Grosso, Av. Fernando Corrêa da Costa, 78060-900, Cuiabá, MT, Brasil \\ ${ }^{2}$ Centro Universitário de Várzea Grande, Av. Dom Orlando Chaves, 2655, 78118-000, Várzea Grande, MT, \\ Brasil \\ ${ }^{3}$ Universidade Federal de Mato Grosso, Programa de Pós-Graduação em Recursos Hídricos, Av. Fernando \\ Corrêa da Costa, 78060-900, Cuiabá, MT, Brasil \\ *Corresponding author: Gisele Fernanda Pereira Assis, e-mail: gisele.fernanda2@gmail.com
}

ASSIS, G. F. P., SILVA, S. P., SILVA, L. F. S., COSTA, R. L., FONSECA, L. W., De-LAMONICA- FREIRE, E. M., FIGUEIREIDO, D. M., TEIXEIRA-OLIVEIRA, M. Inventory of cyanobacteria and microalgae cited for the National Park of the Chapada dos Guimarães (MT) and 80 new records from the region. Biota Neotropica. 18(2): e20170399. http://dx.doi.org/10.1590/1676-0611-BN-2017-0399

\begin{abstract}
Cyanobacteria and microalgae make up a very diverse group and exhibit diverse morphological, biochemical and physiological differences. The knowledge of these organisms that inhabit epicontinental waters in Brazil is extremely heterogeneous in relation to the geographic region and the taxonomic group. Taxonomically there is much more knowledge about the algae of the South and Southeast regions of the country than there is about the algae of the North and Central-West regions. Therefore, this research presents a revised and updated knowledge of the algae that occur in the National Park of the Chapada dos Guimarães (PNCG) and surrounding areas. Our area of study is located in the state of Mato Grosso, in the center-west region of Brazil. The database was obtained from published literature related to taxonomic and ecological inventories, as well as new collections carried out in the area. The collection methods were quite diverse to cover all types of organisms, in plankton, periphyton, filamentous masses, sludge and water accumulated in bromeliads, the new collections are samples collected exclusively in the plankton. They were listed 182 taxa, distributed in 83 genera, 45 families, 11 classes and seven taxonomic divisions. In total, 89 taxa were found in the new samplings, of which nine taxa were already registered in the existing literature, so 80 taxa are new records for the region. A large number of taxa previously mentioned in the literature were not recorded in the sampled environments, most of them composed of Bacillariophyceae, Conjugatophyceae and Euglenophyceae. As well as three species of cyanobacteria of great sanitary importance were registered. Therefore, CGNP has great importance in the maintenance of aquatic biodiversity and to know this community becomes increasingly important to support management measures.
\end{abstract}

Keywords: cerrado, algae, Planktothrix, taxonomy, streams.

\section{Inventário de cianobactérias e microalgas citadas para o Parque Nacional da Chapada dos Guimarães (MT) e 80 novos registros da região}

\footnotetext{
Resumo: As cianobactérias e as microalgas compõem um grupo bastante diversificado e exibem diversas diferenças morfológicas, bioquímicas e fisiológicas. O conhecimento desses organismos que habitam as águas epicontinentais no Brasil é extremamente heterogêneo em relação à região geográfica e ao grupo taxonômico. Taxonomicamente, há muito mais conhecimento sobre as algas das regiões Sul e Sudeste do país do que sobre as algas das regiões Norte e Centro-Oeste. Portanto, esta pesquisa apresenta um conhecimento revisado e atualizado das algas que ocorrem no Parque Nacional da Chapada dos Guimarães (PNCG) e arredores. Nossa área de estudo está localizada no estado de Mato Grosso, na região centro-oeste do Brasil. O banco de dados foi obtido a partir de literatura publicada relacionada a inventários taxonômicos e ecológicos, assim como novas coletas realizadas na área. Os métodos de coletas foram bastante diversos de modo que abrangesse todo tipo de organismos, no plâncton,
} 
perifíton, massas filamentosas, lodo e água acumulada em bromélias, as novas coletas são amostras coletadas exclusivamente no plâncton. Foram listados 182 táxons, distribuídos em 83 gêneros, 45 famílias, 11 classes e sete divisões taxonômicas. No total, 89 táxons foram encontrados nas novas amostragens realizadas, destes, nove táxons, já haviam sido registrados na literatura existente, portanto 80 táxons são novos registros para a região. Um grande número de táxons citados anteriormente na literatura, não foram registrados nos ambientes amostrados, na sua grande maioria constituído por Bacillariophyceae, Conjugatophyceae e Euglenophyceae. Como também foram registradas três espécies de cianobactérias de grande importância sanitária. Sendo assim o PNCG possui grande importância na manutenção da biodiversidade aquática e conhecer essa comunidade torna-se cada vez mais importante para embasar medidas de manejo.

Palavras-chave: cerrado, algas, Planktothrix, taxonomia, riachos.

\section{Introduction}

Cyanobacteria and microalgae comprise a very diverse group of forms, sizes and strategies (Nishimura et al. 2015). However, knowledge about cyanobacteria and microalgae species number and distribution in the epicontinental waters of Brazil is extremely heterogeneous. Taxonomically there is much more knowledge about cyanobacteria and algae of the South and Southeast regions of the country than there is about the algae of the North and Central-West regions (Bicudo \& Menezes 2010), nonetheless Menezes et al (2015) showed increase in species numbers from 2010 to 2015, for state of Mato Grosso.

The Upper Paraguay River Basin (UPB), located in the states of Mato Grosso and Mato Grosso do Sul in the Central-West region of Brazil, is divided into three geomorphologically distinct portions: the plateaus, the depressions and the Pantanal plain. Located in the region of the plateaus is Chapada dos Guimarães National Park (CGNP), an important conservation unit of the Cerrado biome. The CGNP possesses great biological diversity and numerous water sources that form several tributaries of the Pantanal, in particular the Coxipo river, which drains more than 55\% of the total area of CGNP (Lima \& Lima 2009). The CGNP has a fundamental role in conserving the aquatic biota and preserving the water quality of the rivers of the region, whose downstream uses include irrigation, water supply to small farms, fish farming and bathing, as well as the dilution of domestic effluents. Furthermore, the Coxipó river provides more than $30 \%$ of the total water supply of the city of Cuiabá, the capital of Mato Grosso (Gomes-Silva 2015). Even in light of the social and environmental relevance of the rivers that drain CGNP, there have been few studies on its aquatic biota, especially the algal community.

Studies on cyanobacteria and microalgae in state Mato Grosso are scarce, being concentrated in the region of the Pantanal wetland (Freitas \& Loverde-Oliveira 2013; Menezes et al. 2015; Marçal \& LoverdeOliveira 2015), which makes comparative studies difficult. The first studies carried out in the Chapada dos Guimarães region contributed records of 14 species; Closterium gracile, Closterium tumidum and Pleurotaenium maximum cataloged by Borge (1903a); three of the genus Spirogyra and one of the genus Zygogonium cataloged by Dias (1986); and seven species of pigmented Euglenaceae cataloged by Menezes (1986).

Dias (1986) employed a variety of techniques and sampling of plankton from different types of substrates, reporting the importance of the watercourses present in the region. Three years after the publication of Dias (1986), CGNP was created by Federal Decree 97.656 on 12 April, 1989, when several local groups and civil entities began to develop environmental education projects in an attempt to revert the current situation of degradation that was visible in several areas of the region (Teixeira de Oliveira \& Hardoim 2010).

In the same year that CGNP was created, Sophia and Silva (1989) contributed to the knowledge of Conjugatophyceae by recording six species. Among these was Desmidium grevillei (Syn.: Desmidium cylindricum), whose occurrence was not reported again until 25 years later by Fonseca et al. (2014) from the urban area of the municipality of Chapada dos Guimarães. Menezes and Fernandes (1990) are the only ones to have recorded the class Dinophyceae in plankton samples of the region; no other work has since reported the occurrence of this group.

After the work of Menezes \& Fernandes (1990), two coursecompletion papers recorded new occurrences of microalgae in the region, Bazanella (2003; unpublished data), with 32 species of Bacillariophyceae, from plankton samples, and Souza (2004; unpublished data) with 34 taxa of Bacillariophyceae found in phytotelmatic environments of bromeliads. In 2014, Fonseca and collaborators published a list of species from the Quineira river in the Casca river basin, recording 19 species of Zygnematophyceae. The authors did not provide a description of the taxa but presented photographic records of the identified species. As far as we know, there is no synthesis work with registration of cyanobacteria and microalgae for the CGNP region. The data are fragments in articles, dissertations, and course completion papers.

Here we provide an inventory of the cyanobacteria and microalgae recorded in the literature for CGNP and the surrounding area. In doing so, we also reported 80 new records of occurrences from new collections for the park region.

\section{Materials and Methods}

\section{Study site}

The Chapada dos Guimarães National Park (CGNP) is one of the principal conservation and protection units of the Cerrado biome in Brazil. It is located in the Central-West region of the country in the state of Mato Grosso, within the municipalities of Chapada dos Guimarães and Cuiabá. It encompasses approximately 32.630 ha (Lopes et al. 2009), and includes numerous springs, trails, streams, rivers, backwaters and waterfalls, and whose rivers flow into the Cuiabá river, one of the main tributaries of Pantanal (Figure 1). The CGNP is buffered from the intense anthropogenic activities that occur in the region by an Environmental Protection Area (EPA). 


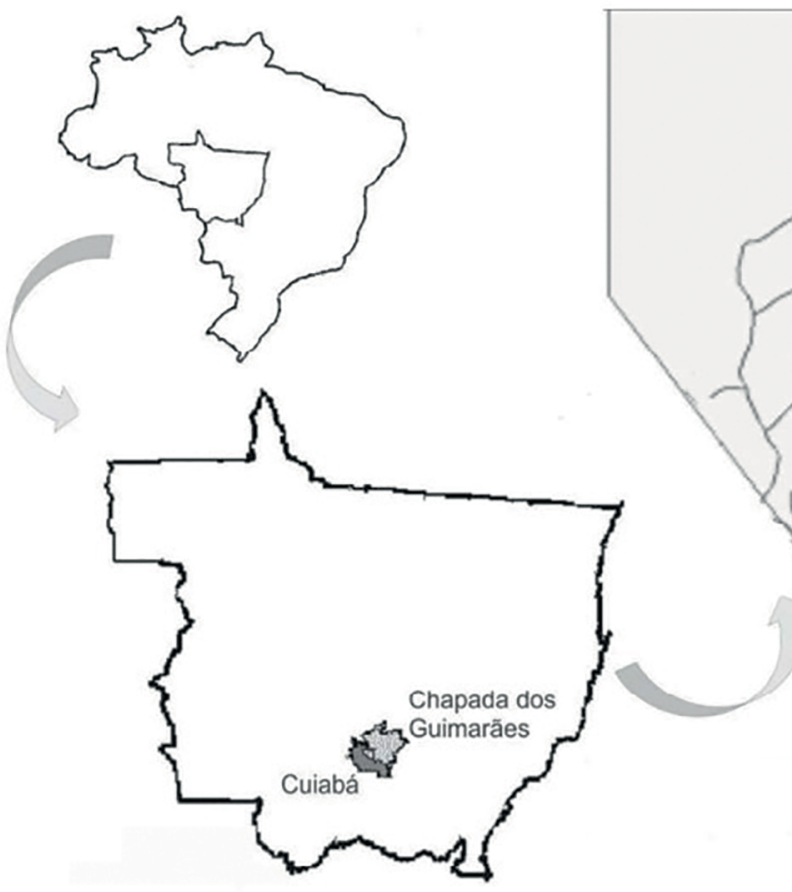

Figure 1. Illustrative map indicating the location of the planktonic algae sampling stations in the watercourses of Chapada dos Guimarães National Park (CGNP) - MT, Brazil, and its surrounding area. (Map adapted from Gomes-Silva 2015).

The region experiences two very different, well-defined seasons with regard to rainfall; a drought (dry season) from May to September, and rains (rainy season) from November to April. According to the Koppen classification, the predominant climate type of the region is included in the categories AW and CW (Padilha Junior \& Nunes 2014).

\section{Data collection}

This research was developed from literature related to taxonomic and ecological inventories, what included articles published in scientific journals, course-completion papers, dissertations and theses that have a list of species of algae from CGNP and the surrounding area. The authors of these works applied the following methods: phytoplankton was collected with a plankton net $(25 \mu \mathrm{m})$; periphyton and sludge was removed from substrates by scraping; filamentous masses and the waters accumulated in bromeliads was collected directly. In all studies, samples were fixed with Transeau solution. Due to the scarcity of work done in the region, all of these sampling methods were considered, however, only taxa identified to species level were included in the current list.

Aiming to broaden the knowledge about cyanobacteria and microalgae taxa already registered for the area, we carried out a taxonomic inventory at ten collection stations in the region (E1 a E10), between August and October of 2013 and in the months of March and June of 2014. We analyzed a total of 44 samples of plankton from Claro, Coxipó, Coxipozinho, Paciência and Mutuca rivers (Figure 1; Table 1).

Samples were collected using a plankton net $(20-\mu \mathrm{m}$ mesh) in the subsurface of the water and preserved in $500 \mathrm{~mL}$ flasks containing $4 \%$ formalin solution (Bicudo \& Menezes 2006). Species identification was based on morphological and metric characteristics of the populations by observing at least 20 individuals of each species on slides with coverslips. Observations were made using a Carl Zeiss optical microscope coupled to an Axiocam 105 color LAB.A1 with maximum resolution of $400 \mathrm{X}$ and a digital camera.

For taxonomic analysis of diatoms, samples were oxidized with heated hydrogen peroxide $\left(\mathrm{H}_{2} \mathrm{O}_{2}\right)$ and hydrochloric acid $(\mathrm{HCl})$, according to the protocol of the European Committee for Standardization. For the observation of frustules, permanent slides were mounted with Naphrax as an inclusion medium. Measurements of length, width, and number of striations in a range of $10 \mu \mathrm{m}$ were taken. Identification of diatoms, when possible, was based on population analysis.

The classification systems used were Komárek (2014) and Rugiero et al. (2015), and the analyzed material was deposited in the liquid phycological collection of the Herbário Central da Universidade Federal de Mato Grosso (UFMTAlgae), campus Cuiabá. 
Table 1. Rivers, sampling station, registration number and geographic coordinates of the planktonic microalgae collection areas of the Chapada dos Guimarães National Park - MT, Brazil, and surrounding area.

\begin{tabular}{lccc}
\hline Rivers & Sampling station & Register N & Geographic coordinates \\
\hline Claro & E1 & UFMTAlgae $01,06,08$ e 11 & $15^{\circ} 18^{\prime} 55,00^{\prime \prime} \mathrm{S}, 055^{\circ} 52^{\prime} 57,00^{\prime \prime} \mathrm{W}$ \\
Claro & E2 & UFMTAlgae 09,13 e 622 & $15^{\circ} 18^{\prime} 36,90^{\prime \prime} \mathrm{S}, 055^{\circ} 53^{\prime} 23,30^{\prime \prime} \mathrm{W}$ \\
Claro & E3 & UFMTAlgae $03,15,657,658,623$ e 624. & $15^{\circ} 18^{\prime} 30,70^{\prime \prime} \mathrm{S}, 055^{\circ} 53^{\prime} 08,60^{\prime \prime} \mathrm{W}$ \\
Claro & E4 & UFMTAlgae $17,626,627$ e 628 & $15^{\circ} 18^{\prime} 52,31^{\prime \prime} \mathrm{S}, 055^{\circ} 52^{\prime} 31,84^{\prime \prime} \mathrm{W}$ \\
Claro & E5 & UFMTAlgae $05,19,20,629,630$ e 631 & $15^{\circ} 19^{\prime} 23,81^{\prime \prime} \mathrm{S}, 055^{\circ} 52^{\prime} 04,15^{\prime \prime} \mathrm{W}$ \\
Coxipozinho & E6 & UFMTAlgae $82,98,276$ e 637 & $15^{\circ} 24^{\prime} 33,5^{\prime \prime} \mathrm{S}, 055^{\circ} 49^{\prime} 54,548^{\prime \prime} \mathrm{W}$ \\
Claro & E7 & UFMTAlgae $97,268,636$ & $15^{\circ} 21^{\prime} 13,151^{\prime \prime} \mathrm{S}, 055^{\circ} 54^{\prime} 52,375^{\prime \prime} \mathrm{W}$ \\
Paciência & E8 & UFMTAlgae 82,98,276,637 & $15^{\circ} 21^{\prime} 13^{\prime \prime} \mathrm{S}, 055^{\circ} 54^{\prime} 52^{\prime \prime} \mathrm{W}$ \\
Mutuca & E9 & UFMTAlgae 83,158,239,284 e 638 & $15^{\circ} 21^{\prime} 52^{\prime \prime} \mathrm{S} ; 055^{\circ} 57^{\prime} 21^{\prime \prime} \mathrm{W}$ \\
Coxipó & E10 & UFMTAlgae $76,96,152,258$ e 635. & $15^{\circ} 22^{\prime} 25^{\prime \prime} \mathrm{S} ; 055^{\circ} 57^{\prime} 50^{\prime \prime} \mathrm{W}$ \\
\hline
\end{tabular}

\section{Results and Discussion}

Our dataset totaled 182 taxa distributed among 83 genera, 45 families, 11 classes and 7 taxonomic divisions. The most representative class in terms of number of families was Bacillariophyceae (16 families), followed by Cyanophyceae ( 9 families). The largest number of taxa was from Bacillariophyceae, with 65 taxa (35.71\%), followed by Conjugatophyceae with 53 taxa (29.12\%), Chlorophyceae with 20 taxa (10.98\%), Cyanophyceae with 15 taxa (8.24\%), and Euglenophyceae with 13 taxa (7.14\%) (Figure 2). A total of 102 taxa were acquired from six published articles and two course-conclusion papers $(56.04 \%)$. Figure 3 presents some representatives of the groups found in new collections carried out in the park.

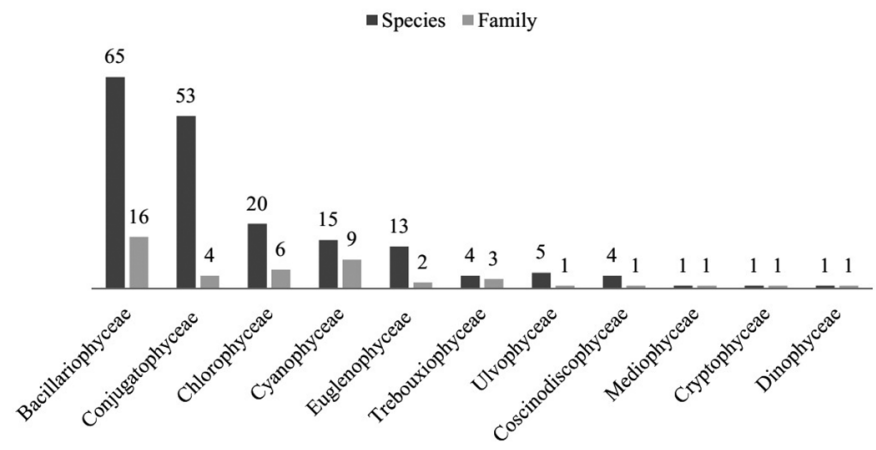

Figure 2. Number of family and species distributed by classes.

In total, 89 taxa were found in the new sampling performed in the study area, of which nine (Eunotia camelus, Gomphonema gracile, Aulacoseira granulata, Bambusina borreri, Cosmarium pseudoconnatum, Cosmarium obsoletum, Cosmarium contractum and Micrasterias papillifera) had already been recorded in the literature and 80 representing new records for the region (43.95\%) (Table 2). Ninety-three previously cited taxa were not recorded in the sampled environments, most of which were of Bacillariophyceae (45 taxa), Conjugatophyceae (23 taxa) and Euglenophyceae ( 9 taxa). The high representation of Bacillariophyceae in the literature is due to the different types of environments sampled that favor the development of diatomaceous species.
Bacillariophyceae are one of the most common and dominant groups in both plankton and periphyton (Stevenson 1996; Bellinger \& Sigee 2010), due to their variety of forms that allows colonization of different types of substrates. They also have high species richness, play an important role as primary producers and are considered bioindicators of the trophic state of these aquatic environments (Bellinger \& Sigee 2010). With regard to diatom diversity in general, the Neotropics is considered quite rich compared to the well-studied temperate regions of Europe and North America, and each new published flora results in dozens of new proposed taxa, some of which are particularly endemic (Cavalcante et al. 2014), but no endemism was found in CGNP for this group of algae. Although the occurrence of diatoms is related to water flow and success of substrate colonization, the ability of these organisms to survive in these environments depends on factors associated with their distribution, such as nutrients, light, temperature, among others (Reynolds et al. 2006).

The families with the greatest species richness were Desmidiaceae, with 35 taxa (19.23\%), and Eunotiaceae, with 19 taxa (10.44\%). Desmids are remarkable because of the great diversity of species that are distributed in a variety of different environments, such as plankton, periphyton and metaphyton (Menezes et al. 2011), and their preference for acidic environments - oligotrophic to eutrophic (Bicudo \& Menezes 2006), characteristics that favor the development of the group in the region with its crystalline waters, rivers with waterfalls and preserved riparian vegetation. As with desmids, representatives of the family Eunotiaceae are favored by acidic and nutrient poor environments (Round et al. 1990), prevailing conditions in the rivers and streams sampled in this study (Gomes-Silva 2015).

There was an increase in the records of cyanobacteria in the region, with two species described by Bazanella (2003; unpublished data), Kamptonema proteus and Pseudanabaena galeata, and 13 species found in current collections (Cylindrospermopsis raciborskii, Asterocapsa submersa, Chroococcus dispersus, Chroococcus minor, Snowella lacustris, Komvophoron crassum, Komvophoron schmidlei, Merismopedia tenuissima, Planktothrix agardhii, Microcystis aeruginosa, Sphaerocavum brasiliense, Lyngbya major and Pseudanabaena galeata). Three cyanotoxin-producing species of major public health importance were recorded in the study area, Cylindrospermopsis raciborskii, Planktothrix agardhii and 

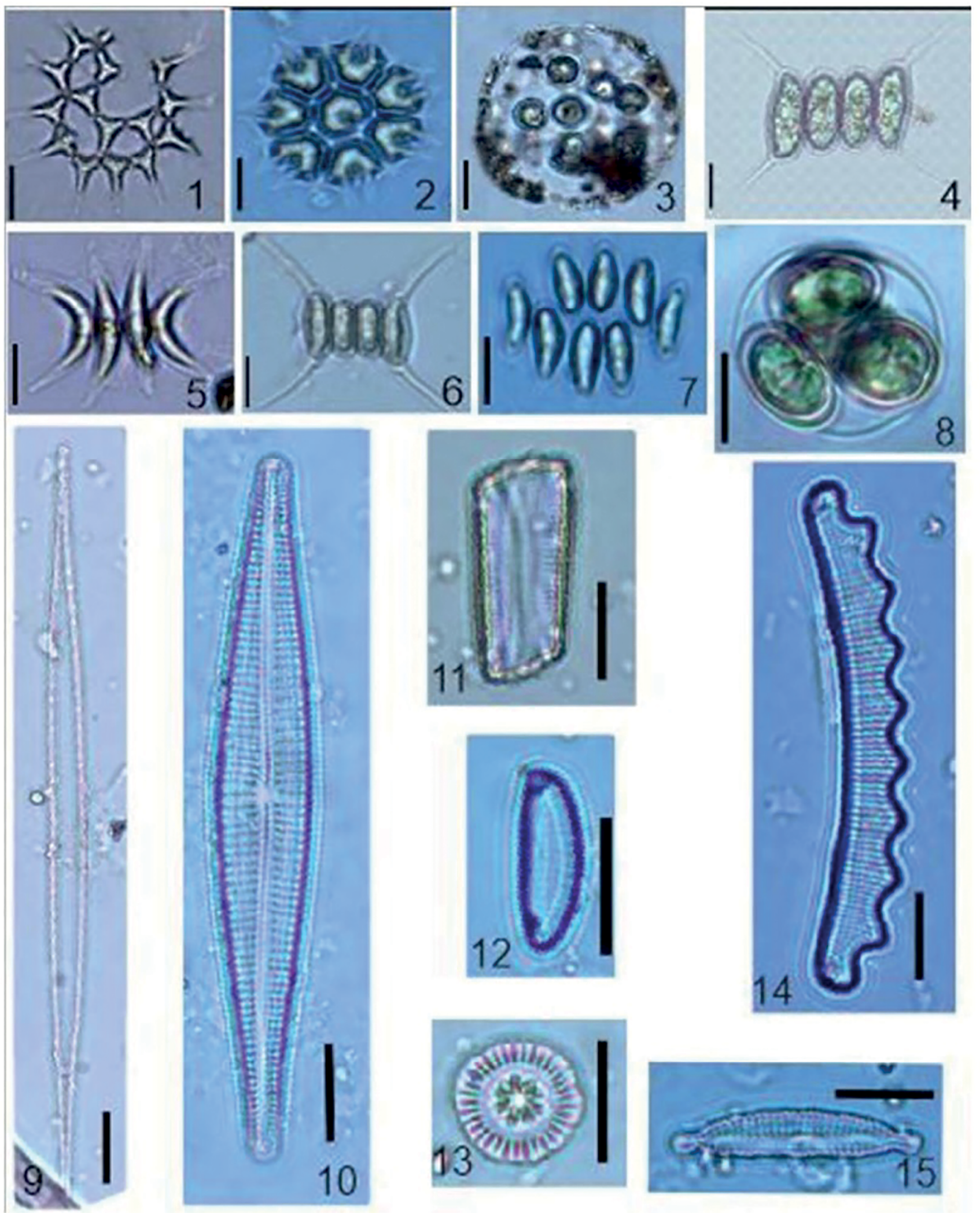

Figure 3. 1. Monactinus simplex, 2. Stauridium tetras, 3. Sphaerocystis planctonica, 4. Desmodesmus communis, 5. Acutodesmus acuminatus, 6. Desmodesmus opoliensis, 7. Scenedesmus obtusus, 8. Oocystis borgei, 9. Stenopterobia delicatissima; 10. Gomphonema gracile; 11-12. Eunotia botuliformis; 13. Discostella pseudostelligera; 14. Eunotia georgii; 15. Encyonema angustecapitatum. Scale bar: $10 \mu \mathrm{m}$. 
Assis, G.F.P. et al.

Table 2. List of microalgae recorded in the Chapada dos Guimarães National Park and surroundings, Mato Grosso, Central-West Brazil. * new records for the Chapada dos Guimarães National Park and surroundings.

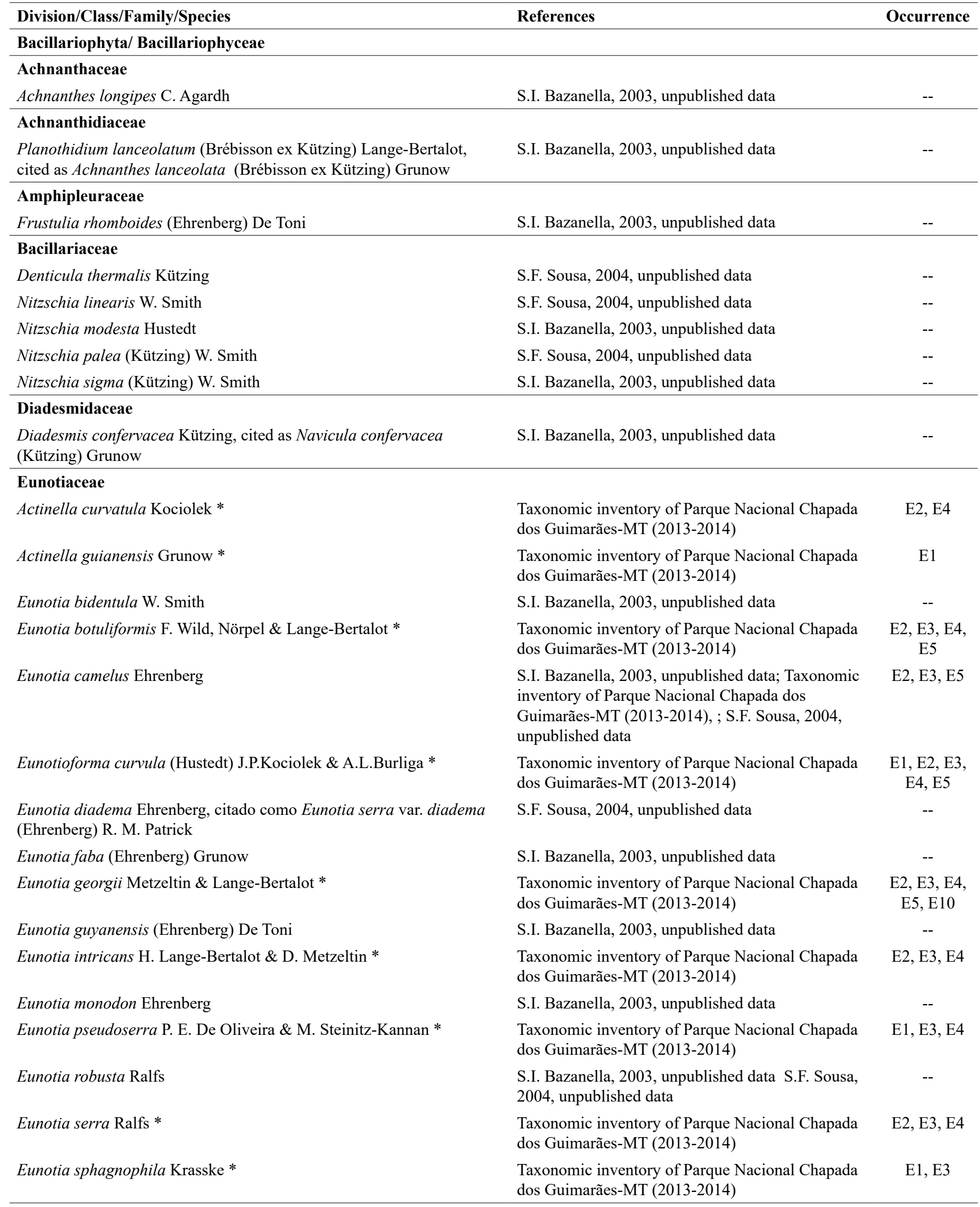


Continued Table 2.

\begin{tabular}{ll}
\hline Division/Class/Family/Species & References \\
\hline Eunotia subrobusta Hustedt * & $\begin{array}{l}\text { Taxonomic inventory of Parque Nacional Chapada } \\
\text { dos Guimarães-MT (2013-2014) }\end{array}$ \\
Eunotia trigibba Hustedt * & $\begin{array}{l}\text { Taxonomic inventory of Parque Nacional Chapada } \\
\text { dos Guimarães-MT (2013-2014) }\end{array}$ \\
Eunotia triodon Ehrenberg & S.F. Sousa, 2004, unpublished data
\end{tabular}

\section{Fragilariaceae}

Fragilaria crotonensis Kitton

Fragilaria rolandschmidtii Metzeltin \& Lange-Bertalot*

Fragilariforma virescens (Ralfs) D. M. Williams \& Round, citado como Fragilaria virescens Ralfs

\author{
Gomphonemataceae \\ Encyonema angustecapitatum Krammer * \\ Encyonopsis frequentis Krammer * \\ Gomphonema angustatum (Kützing) Rabenhorst \\ Gomphonema brasiliense Grunow \\ Gomphonema gracile Ehrenberg *
}

Gomphonema martini F. Fricke

Gomphonema olivaceum (Hornemann) Brébisson

Gomphonema parvulum (Kützing) Kützing

Gomphonema turris Ehrenberg citado como Gomphonema augur var. turris (Ehrenberg) Lange-Bertalot

Placoneis pseudanglica E. J. Cox *

\section{Naviculaceae}

Navicula cryptocephala Kützing *

Navicula oblonga (Kützing) Kützing

Navicula cuspidata (Kutzing) Kutzing

\section{Neidiaceae}

Neidium affine var. ceylonicum (Skvortsov) Reimer *

\section{Pinnulariaceae}

Pinnularia gibba var. sancta (Grunow ex Cleve) F. Meister

Pinnularia lata (Brébisson) W. Smith

Pinnularia mayeri Krammer, citado como Pinnularia braunii var. amphicephala (Ant. Mayer) Hustedt

Pinnularia viridis (Nitzsch) Ehrenberg

\section{Rhopalodiaceae}

Epithemia adnata (Kützing) Brébisson citado como Epithemia zebra (Ehrenberg) Ehrenberg

Epithemia argus (Ehrenberg) Kützing

Epithemia argus var. longicornis (Ehrenberg) Grunow
S.I. Bazanella, 2003, unpublished data

Taxonomic inventory of Parque Nacional Chapada E2, E3 dos Guimarães-MT (2013-2014)

S.I. Bazanella, 2003, unpublished data
Taxonomic inventory of Parque Nacional Chapada dos Guimarães-MT (2013-2014)

Taxonomic inventory of Parque Nacional Chapada dos Guimarães-MT (2013-2014)

S.I. Bazanella, 2003, unpublished data

S.I. Bazanella, 2003, unpublished data

Taxonomic inventory of Parque Nacional Chapada dos Guimarães-MT (2013-2014); S.F. Sousa, 2004, unpublished data

S.F. Sousa, 2004, unpublished data

S.I. Bazanella, 2003, unpublished data

S.F. Sousa, 2004, unpublished data

S.F. Sousa, 2004, unpublished data

Taxonomic inventory of Parque Nacional Chapada dos Guimarães-MT (2013-2014)

Taxonomic inventory of Parque Nacional Chapada dos Guimarães-MT (2013-2014)

S.I. Bazanella, 2003, unpublished data

S.I. Bazanella, 2003, unpublished data

Taxonomic inventory of Parque Nacional Chapada dos Guimarães-MT (2013-2014)

$\mathrm{E} 1, \mathrm{E} 2, \mathrm{E} 3$, E4, E10

$--$

$-$

$--$

$--$

E2, E3
E2, E3

E2, E4, E5

$--$

$--$
$\mathrm{E} 3, \mathrm{E} 4$

, E4

$-$

E10

E3

S.F. Sousa, 2004, unpublished data

S.F. Sousa, 2004, unpublished data

S.F. Sousa, 2004, unpublished data

S.I. Bazanella, 2003, unpublished data

$--$

$-$

S.F. Sousa, 2004, unpublished data

S.I. Bazanella, 2003, unpublished data

S.F. Sousa, 2004, unpublished data

(1)


Continued Table 2.

\begin{tabular}{l}
\hline Division/Class/Family/Species \\
Surirellaceae \\
Iconella curvula (Smith) Ruck \& Nakov cited as Stenopterobia curvula \\
(W. Smith) Krammer* \\
Iconella delicatissima (F.W.Lewis) Ruck \& Nakov cited as \\
Stenopterobia delicatissima (F. W. Lewis) Brébisson ex Van Heurck* \\
Surirella elegans Ehrenberg \\
Iconella guatimalensis (Ehrenberg) Ruck \& Nakov cited as Surirella \\
guatimalensis Ehrenberg \\
Surirella minuta Brébisson ex Kützing, citado como Surirella ovata \\
Kützing \\
Surirella robusta Ehrenberg
\end{tabular}

\section{Stauroneidaceae}

Craticula cuspidata (Kützing) D. G. Mann, citada como Navicula cuspidata (Kützing) Kutzing

Stauroneis deperdita Manguin

Tabellariaceae
Diatoma anceps (Ehrenberg) Kirchner
Diatoma vulgaris Bory
Tabellaria fenestrata (Lyngbye) Kützin

\section{Ulnariaceae}

Hannaea arcus (Ehrenberg) R. M. Patrick, citado como Ceratoneis arcus (Ehrenberg) Kütizing

Ulnaria capitata (Ehrenberg) Compère, citado como Synedra capitata Ehrenberg

\section{Bacillariophyta/Coscinodiscophyceae}

Aulacoseiraceae

Aulacoseira distans (Ehrenberg) Simonsen

Aulacoseira granulata (Ehrenberg) Simonsen

Aulacoseira herzogii (Lemmermann) Simonsen

Aulacoseira italica (Ehrenberg) Simonsen

\section{References}

Occurrence

Taxonomic inventory of Parque Nacional Chapada dos Guimarães-MT (2013-2014)

Taxonomic inventory of Parque Nacional Chapada dos Guimarães-MT (2013-2014)

S.I. Bazanella, 2003, unpublished data

$\mathrm{E} 1, \mathrm{E} 2, \mathrm{E} 4$

S.I. Bazanella, 2003, unpublished data

S.I. Bazanella, 2003, unpublished data

S.I. Bazanella, 2003, unpublished data

S.I. Bazanella, 2003, unpublished data

S.I. Bazanella, 2003, unpublished data

S.I. Bazanella, 2003, unpublished data

S.I. Bazanella, 2003, unpublished data

S.I. Bazanella, 2003, unpublished data

S.I. Bazanella, 2003, unpublished data

S.I. Bazanella, 2003, unpublished data
$--$

$-$

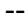

$--$

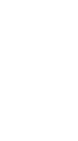

\section{Bacillariophyta/Mediophyceae}

\section{Stephanodiscaceae}

Discostella pseudostelligera (Hustedt) Houk \& Klee *

Taxonomic inventory of Parque Nacional Chapada

$\mathrm{E} 1, \mathrm{E} 2, \mathrm{E} 4$ dos Guimarães-MT (2013-2014);

Chlorophyta/Chlorophyceae

\section{Hydrodictyaceae}

Lacunastrum gracillimum (West \& G. S. West) H. McManus *

Taxonomic inventory of Parque Nacional Chapada

Monactinus simplex (Meyen) Corda * dos Guimarães-MT (2013-2014)

Taxonomic inventory of Parque Nacional Chapada

E4, E5 dos Guimarães-MT (2013-2014)

Stauridium tetras (Ehrenberg) E. Hegewald*

Taxonomic inventory of Parque Nacional Chapada dos Guimarães-MT (2013-2014)

Microsporaceae

Microspora membranacea Wang

S.F. Sousa, 2004, unpublished data 
Continued Table 2.

\begin{tabular}{l} 
Division/Class/Family/Species \\
\hline Neochloridaceae \\
Golenkinia radiata Chodat * \\
Scenedesmaceae \\
Acutodesmus acuminatus (Lagerheim) P. M. Tsarenko * \\
Coelastrum proboscideum Bohlin * \\
Coelastrum sphaericum Nägeli, citado como Coelastrum verrucosum \\
(Reinsch) Reinsch *
\end{tabular}

Desmodesmus armatus var. bicaudatus (Guglielmetti) E. Hegewald *

Desmodesmus communis (E. Hegewald) E. Hegewald *

References

Occurrence

Taxonomic inventory of Parque Nacional Chapada

E9

dos Guimarães-MT (2013-2014)

Taxonomic inventory of Parque Nacional Chapada

E9

dos Guimarães-MT (2013-2014)

Taxonomic inventory of Parque Nacional Chapada

dos Guimarães-MT (2013-2014)

Taxonomic inventory of Parque Nacional Chapada

dos Guimarães-MT (2013-2014)

Taxonomic inventory of Parque Nacional Chapada dos Guimarães-MT (2013-2014)

Taxonomic inventory of Parque Nacional Chapada dos Guimarães-MT (2013-2014)

Desmodesmus opoliensis (P.G. Richter) E. Hegewald *

Taxonomic inventory of Parque Nacional Chapada dos Guimarães-MT (2013-2014)

Dimorphococcus lunatus A. Braun *

Taxonomic inventory of Parque Nacional Chapada dos Guimarães-MT (2013-2014)

Pectinodesmus javanensis (Chodat) E. Hegewald, C. Bock \& Krienitz * Taxonomic inventory of Parque Nacional Chapada dos Guimarães-MT (2013-2014)

Scenedesmus obtusus Meyen *

Taxonomic inventory of Parque Nacional Chapada dos Guimarães-MT (2013-2014)

Westella botryoides (West) De Wildeman *

Taxonomic inventory of Parque Nacional Chapada dos Guimarães-MT (2013-2014)

\section{Selenastraceae}

Ankistrodesmus arcuatus Korshikov*

Taxonomic inventory of Parque Nacional Chapada dos Guimarães-MT (2013-2014)

Messastrum gracile (Reinsch) T.S.Garcia, citado como Ankistrodesmus gracilis (Reinsch) Korshikov *

Taxonomic inventory of Parque Nacional Chapada dos Guimarães-MT (2013-2014)

Monoraphidium contortum (Thuret) Komárková-Legnerová *

Taxonomic inventory of Parque Nacional Chapada dos Guimarães-MT (2013-2014)

Monoraphidium intermedium Hindák *

Taxonomic inventory of Parque Nacional Chapada dos Guimarães-MT (2013-2014)

\section{Sphaerocystidaceae}

Sphaerocystis planctonica (Korshikov) Bourrelly *

Taxonomic inventory of Parque Nacional Chapada dos Guimarães-MT (2013-2014)

\section{Chlorophyta/Trebouxiophyceae}

\section{Chlorellaceae}

Mucidosphaerium pulchellum (H.C.Wood) C.Bock, Proschold \&

Taxonomic inventory of Parque Nacional Chapada

\section{Oocystaceae}

Oocystis borgei J. W. Snow *

Taxonomic inventory of Parque Nacional Chapada

Trebouxiophyceae Família incertae sedis

Lemmermannia komarekii (Hindák) C.Bock \& Krienitz *

Taxonomic inventory of Parque Nacional Chapada

E9

Lemmermannia triangularis (Chodat) C. Bock \& Krienitz *

dos Guimarães-MT (2013-2014)

Taxonomic inventory of Parque Nacional Chapada

E9 dos Guimarães-MT (2013-2014) 
Continued Table 2.

Division/Class/Family/Species

Chlorophyta/Ulvophyceae

\section{Ulotrichaceae}

Ulothrix bipyrenoidosa F. E. Fritsch \& M. F. Rich

Ulothrix implexa (Kützing) Kützing

Ulothrix limnetica Lemmermann

Ulothrix tenerrima (Kützing) Kützing, citado como Ulothrix variabilis

Kützing

Ulothrix zonata (F. Weber \&amp; Mohr) Kützing

Charophyta/Conjugatophyceae

Closteriaceae

Closterium acutum var. variabile (Lemmermann) Willi Krieger *

Closterium cornu Ehrenberg ex Ralfs

Closterium costatum var. borgei (Willi Krieger) Ruzicka *

Closterium ehrenbergii Meneghini ex Ralfs

Closterium gracile Brébisson ex Ralfs

Closterium intermedium Ralfs

Closterium jenneri var. cynthia (De Notaris) Petlovany, citado como

Closterium cynthia De Notaris

Closterium juncidum Ralfs

Closterium moniliferum var. moniliferum f. gracile Kurt Förster *

Closterium navicula (Brébisson) Lütkemüller *

Closterium tumidum L. N. Johnson

Closterium venus Kützing ex Ralfs

\section{Desmidiaceae}

Actinotaenium cruciferum var. cruciferum (De Bary) Teiling *

Actinotaenium globosum (Bulnheim) Kurt Förster ex Compère *

Actinotaenium silvae-nigrae (Rabanus) Kouwets \& Coesel, citado como Penium silvae-nigrae Rabanus

Actinotaenium wollei (West \& G. S. West) Teiling

Bambusina borreri (Ralfs) Cleve, cited in ( ${ }^{1}$ ) as Bambusina brebissonii

Kützing ex Kützing ${ }^{1}$

Cosmarium circulare Reinsch, citado em freitas como C. candianum*

Cosmarium connatum Brébisson ex Ralfs

Cosmarium contractum $\mathrm{O}$. Kirchner

Cosmarium furcatospermum West \& G. S. West

Cosmarium obsoletum (Hantzsch) Reinsch
References

Occurrence

S.F. Sousa, 2004, unpublished data

$--$

S.F. Sousa, 2004, unpublished data

S.F. Sousa, 2004, unpublished data

S.F. Sousa, 2004, unpublished data

$--$

S.F. Sousa, 2004, unpublished data

Taxonomic inventory of Parque Nacional Chapada dos Guimarães-MT (2013-2014)

Fonseca et al. (2014)

Taxonomic inventory of Parque Nacional Chapada dos Guimarães-MT (2013-2014)

Fonseca et al. (2014)

Borge (1903a)

S.F. Sousa, 2004, unpublished data

Fonseca et al. (2014)

S.F. Sousa, 2004, unpublished data

Taxonomic inventory of Parque Nacional Chapada dos Guimarães-MT (2013-2014)

Taxonomic inventory of Parque Nacional Chapada dos Guimarães-MT (2013-2014)

Borge (1903a)

S.F. Sousa, 2004, unpublished data

Taxonomic inventory of Parque Nacional Chapada dos Guimarães-MT (2013-2014)

Taxonomic inventory of Parque Nacional Chapada dos Guimarães-MT (2013-2014)

S.F. Sousa, 2004, unpublished data

Fonseca et al. (2014)

Taxonomic inventory of Parque Nacional Chapada dos Guimarães-MT (2013-2014), ${ }^{1}$ Sophia \& Silva (1989)

Taxonomic inventory of Parque Nacional Chapada dos Guimarães-MT (2013-2014)

Fonseca et al. (2014)

Taxonomic inventory of Parque Nacional Chapada dos Guimarães-MT (2013-2014); Fonseca et al. (2014)

Fonseca et al. (2014)

Taxonomic inventory of Parque Nacional Chapada dos Guimarães-MT (2013-2014); Fonseca et al. (2014) 
Continued Table 2.

\begin{tabular}{l} 
Division/Class/Family/Species \\
\hline Cosmarium pseudoconnatum Nordstedt
\end{tabular}

\section{Cosmarium
Gerloff *}

Cosmarium quadratum Ralfs ex Ralfs *

Cosmarium quadrum P. Lundell

Desmidium grevillei (Kützing ex Ralfs) De Bary, citado como Desmidium cylindricum Greville

Desmidium swartzii C. Agardh ex Ralfs *

Euastrum brasiliense Borge

Euastrum sinuosum var. sinuosum Archerk *

Hyalotheca dissiliens Brébisson ex Ralfs

Hyalotheca dissiliens (Smith) Bréb. var. hians Wolle

Micrasterias decemdentata (Nägeli) W. Archer

Micrasterias denticulata Brébisson ex Ralfs *

Micrasterias laticeps var. acuminata Willi Krieger *

Micrasterias papillifera Brébisson ex Ralfs

Micrasterias rotata Ralfs *

Micrasterias torreyi var. nordstedtiana (Hyeronimus) Schmidle *

Micrasterias truncata Brébisson ex Ralfs *

Pleurotaenium maximum (Reinsch) P. Lundell

Sphaerozosma desmidiiforme Borge citado como Spondylosium desmidiiforme (Borge) G.S.West

Spondylosium pulchrum (Bail.) Archer var. pulchrum, in Pritch

Staurastrum margaritaceum Meneghini ex Ralfs

Staurastrum excavatum West \& G. S. West*

Staurodesmus convergens (Ehrenberb g ex Ralfs) S. Lillieroth *

Staurodesmus validus (West \& G. S. West) Thomasson

Teilingia granulata (J.Roy \& Bisset) Bourrelly

\section{Mesotaeniaceae}

Netrium digitus (Brébisson ex Ralfs) Itzigsohn \& Rothe

Netrium parvum (Borge) Petlovany *

\section{References}

Taxonomic inventory of Parque Nacional Chapada

dos Guimarães-MT (2013-2014); Fonseca et al.

(2014)

Taxonomic inventory of Parque Nacional Chapada dos Guimarães-MT (2013-2014)

Taxonomic inventory of Parque Nacional Chapada dos Guimarães-MT (2013-2014)

Fonseca et al. (2014)

Sophia \& Silva (1989) Fonseca et al. (2014)

Taxonomic inventory of Parque Nacional Chapada dos Guimarães-MT (2013-2014)

Fonseca et al. (2014)

Taxonomic inventory of Parque Nacional Chapada dos Guimarães-MT (2013-2014)

Taxonomic inventory of Parque Nacional Chapada dos Guimarães-MT (2013-2014); Fonseca et al. (2014)

Sophia \& Silva (1989)

Fonseca et al. (2014)

Taxonomic inventory of Parque Nacional Chapada dos Guimarães-MT (2013-2014)

Taxonomic inventory of Parque Nacional Chapada dos Guimarães-MT (2013-2014)

Taxonomic inventory of Parque Nacional Chapada dos Guimarães-MT (2013-2014); Fonseca et al. (2014)

Taxonomic inventory of Parque Nacional Chapada dos Guimarães-MT (2013-2014)

Taxonomic inventory of Parque Nacional Chapada dos Guimarães-MT (2013-2014)

Taxonomic inventory of Parque Nacional Chapada dos Guimarães-MT (2013-2014)

Borge (1903a)

Sophia \& Silva (1989)

Sophia \& Silva (1989)

Fonseca et al. (2014)

Taxonomic inventory of Parque Nacional Chapada dos Guimarães-MT (2013-2014)

Taxonomic inventory of Parque Nacional Chapada dos Guimarães-MT (2013-2014)

Occurrence

E9

E1, E5

E1

$--$

$-$

E3, E4

$--$

E1, E2, E4, E5

$\mathrm{E} 1, \mathrm{E} 2$

$--$

$--$

E4

E1, E2

$\mathrm{E} 1, \mathrm{E} 3, \mathrm{E} 4$

E1, E3

E3

E1, E5

Fonseca et al. (2014)

Sophia \& Silva (1989)

Fonseca et al. (2014)

Taxonomic inventory of Parque Nacional Chapada dos Guimarães-MT (2013-2014) 
Continued Table 2.

Division/Class/Family/Species

References

Occurrence

Zygnemataceae

Spirogyra irregularis Nägeli ex Kützing

Dias (1986)

$-$

Spirogyra macrospora (C. B. Rao) Krieger

Dias (1986)

Spirogyra neglecta (Hassall) Kützing

Dias (1986)

Zygogonium ericetorum Kützing

Dias (1986)

Cryptophyta/Cryptophyceae

\title{
Cryptomonadaceae
}

Cryptomonas marssonii Skuja *

Taxonomic inventory of Parque Nacional Chapada

E8, E9 dos Guimarães-MT (2013-2014)

Cyanobacteria/Cyanophyceae

Aphanizomenonaceae

Cylindrospermopsis raciborskii (Woloszynska) Seenayya \& Subba

Taxonomic inventory of Parque Nacional Chapada

\section{Chroococcaceae}

Asterocapsa submersa Azevedo, Sant'Anna, Senna, Komárek \&

Taxonomic inventory of Parque Nacional Chapada

Komárková * dos Guimarães-MT (2013-2014)

Chroococcus dispersus (Keissler) Lemmermann *

Taxonomic inventory of Parque Nacional Chapada dos Guimarães-MT (2013-2014)

Chroococcus minor (Kützing) Nägeli *

Taxonomic inventory of Parque Nacional Chapada dos Guimarães-MT (2013-2014)

\section{Coelosphaeriaceae}

Snowella lacustris (Chodat) Komárek \& Hindák *

Taxonomic inventory of Parque Nacional Chapada

dos Guimarães-MT (2013-2014)

\section{Gomontiellaceae}

Komvophoron crassum (Vozzhennikova) Anagnostidis \& Komárek *

Taxonomic inventory of Parque Nacional Chapada

Komvophoron schmidlei (Jaag) Anagnostidis \& Komárek * dos Guimarães-MT (2013-2014)

Taxonomic inventory of Parque Nacional Chapada

E1, E4, E10 dos Guimarães-MT (2013-2014)

\section{Merismopediaceae}

Merismopedia tenuissima Lemmermann *

Taxonomic inventory of Parque Nacional Chapada

\section{Microcoleaceae}

Planktothrix agardhii (Gomont) Anagnostidis \& Komárek * dos Guimarães-MT (2013-2014)

\section{Microcystaceae}

Microcystis aeruginosa (Kützing) Kützing *

Taxonomic inventory of Parque Nacional Chapada

\author{
Sphaerocavum brasiliense De Azevedo \& C.L.Sant' Anna*
}

Taxonomic inventory of Parque Nacional Chapada

\section{Oscillatoriaceae}

Lyngbya major Meneghini ex Gomont*

Taxonomic inventory of Parque Nacional Chapada

Oscillatoria proteus Skuja

S.I. Bazanella, 2003, unpublished data

\section{Pseudanabaenaceae}

Pseudanabaena galeata Böcher *

Taxonomic inventory of Parque Nacional Chapada dos Guimarães-MT (2013-2014)

E8, E9, E10

Pseudanabaena limnetica (Lemmermann) Komárek, citado como S.I. Bazanella, 2003, unpublished data 
Continued Table 2.

\begin{tabular}{|c|c|c|}
\hline Division/Class/Family/Species & References & Occurrence \\
\hline \multicolumn{3}{|l|}{ Euglenophyta/Euglenophyceae } \\
\hline \multicolumn{3}{|l|}{ Euglenaceae } \\
\hline Trachelomonas volvocinopsis Svirenko * & $\begin{array}{l}\text { Taxonomic inventory of Parque Nacional Chapada } \\
\text { dos Guimarães-MT (2013-2014) }\end{array}$ & E9 \\
\hline \multicolumn{3}{|l|}{ Phacaceae } \\
\hline $\begin{array}{l}\text { Lepocinclis globulus Perty, citado como Lepocinclis ovum (Ehrenberg) } \\
\text { Lemmermann var. globula (Perty) Lemmermann }\end{array}$ & Menezes (1986) & -- \\
\hline Lepocinclis ovum var. dimidio-minor (Deflandre) Conrad. & Menezes (1986) & -- \\
\hline $\begin{array}{l}\text { Lepocinclis ovum (Ehrenberg) Lemmermann, citado como Lepocinclis } \\
\text { ovum (Ehrenberg) Lemmermann var. ovum }\end{array}$ & Menezes (1986) & -- \\
\hline Phacus longicauda (Ehrenberg) Dujardin * & $\begin{array}{l}\text { Taxonomic inventory of Parque Nacional Chapada } \\
\text { dos Guimarães-MT (2013-2014) }\end{array}$ & $\begin{array}{l}\mathrm{E} 1, \mathrm{E} 2, \mathrm{E} 3, \\
\mathrm{E} 4, \mathrm{E} 5\end{array}$ \\
\hline Phacus orbicularis K. Hübner & Menezes (1986) & 0 \\
\hline Phacus pleuronectes (O. F. Müller) Nitzsch ex Dujardin * & $\begin{array}{l}\text { Taxonomic inventory of Parque Nacional Chapada } \\
\text { dos Guimarães-MT (2013-2014) }\end{array}$ & E3, E5, E9 \\
\hline Phacus pusillus Lemmermann & Menezes (1986) & -- \\
\hline Phacus raciborskii Drezepolski & Menezes (1986) & -- \\
\hline \multicolumn{3}{|l|}{ Miozoa/Dinophyceae } \\
\hline \multicolumn{3}{|l|}{ Peridiniopsidaceae } \\
\hline
\end{tabular}

Microcystis aeruginosa, although records of the occurrence the toxic species were not expected. These species are successful in shallow eutrophic ecosystems (Bittencourt-Oliveira et al. 2014), different from the region in question, which has environments considered as oligotrophic and slightly acidic (Teixeira-Oliveira \& Hardoim 2010). The occurrence of these species may be associated with the presence of mammalian waste from the region, or even the existence of small farms and the very intense tourism that occurs in the studied rivers. This was a concern previously reported by Dias in 1986, and needs to be monitored, including in areas associated with effluents from the urban area of Chapada dos Guimarães, located upstream of the CGNP.

The high concentration of these species in aquatic ecosystems can trigger the production of toxins such as cylindrospermopsin and microcystin, resulting in health problems such as diarrhea, vomiting and weakness, and reaching organs such as the kidneys and the liver. Costa et al. (2017) reports the existence of these potentially toxic species in fishponds in the state of Mato Grosso. Currently in Brazil, microcystins were included as a monitoring parameter in Portaria 1469 legislation (Brasil 2000) and have been used in water quality control ever since (Guerra et al. 2015).

The CGNP is of vital importance to the maintenance of aquatic biodiversity of the region, and understanding this community is becoming increasingly important for management measures. National parks are required to undertake inventories of their biodiversity, which serve as sources of reference data (Moresco \& Rodrigues 2013). The Cerrado network and the Pró Centro-Oeste-REMISA network have developed projects for the diagnosis, analysis and synthesis of biodiversity data concerning CGNP. The studies of these projects have supported the exchange of technical information, as well as decisionmaking by the central manager of CGNP for the management and conservation of the aquatic ecosystems of the park.

The results from this work demonstrate an important increase of 80 species in the list of taxa existing in the region, an important advance for the knowledge of the ficology in the Center-West region of Brazil in recent years. However, 93 previously recorded taxa in the region were not sampled again. We believe that part of this loss in our studies is due to the exclusive collect made in the plankton and that other works that cover a greater number of habitats are important to evidence if there was loss of diversity.

Reducing the time interval between species inventories in watercourses is important to monitor the effect of land occupation and the impact of tourism on local biodiversity. For this, the monitoring of species in the region becomes a tool that is closely related to the integrity of the aquatic ecosystems existing in the park and its surroundings. We emphasize the importance of specialized taxonomist professionals for a better understanding of these microorganisms. Due to the great extension 
of water drainage of the CGNP, we conclude with the certainty that there are still gaps to be filled regarding studies of cyanobacteria and microalgae for the region studied.

\section{Acknowledgements}

We thank Conselho Nacional de Desenvolvimento Científico e Tecnológico- $\mathrm{CNPq}$ and the Fundação de Amparo à Pesquisa do Estado de Mato Grosso - FAPEMAT for financial support through the projects: Rede Pró Centro-Oeste-REMISA (Projeto CNPq n ${ }^{\circ}$ 564617/2010-5 and Projeto FAPEMAT $\left.n^{\circ} 232949 / 2011\right)$, coordinated by Eliana Freire Gaspar de Carvalho Dores, and Rede ComCerrado/Núcleo UFMT, processo ${ }^{\circ} 563134 / 2010$. Lívia Franco da Costa aided in the identification of diatoms, and Denise de Campos Bicudo graciously provided assistance in the production of the their permanent slides.

We thank Coordenação de Aperfeiçoamento de Pessoal de Nível Superior-CAPES for granting a PNPD grant to Daniela Maimoni de Figueiredo of the Programa de Pós-Graduação em Recursos Hídricos.

\section{Author Contributions}

Gisele Fernanda Pereira Assis: Substantial contribution in the concept and design of the study; Contribution to data collection; Contribution to data analysis and interpretation; Contribution to manuscript preparation; Contribution to critical revision, adding intelectual content.

Samiris Pereira da Silva: Contribution to data collection; Contribution to data analysis and interpretation; Contribution to manuscript preparation.

Ludmylla Fernanda de Siqueira Silva: Contribution to data collection; Contribution to data analysis and interpretation.

Regiane Luiza da Costa: Contribution to data collection; Contribution to data analysis and interpretation.

Luany Weiler da Fonseca: Contribution to data collection.

Ermelinda Maria De-Lamonica-Freire: Contribution to data collection; Contribution to critical revision, adding intelectual content.

Daniela Maimoni de Figueireido: Contribution to data collection; Contribution to critical revision, adding intelectual content.

Márcia Teixeira de Oliveira: Substantial contribution in the concept and design of the study; Contribution to data collection; Contribution to data analysis and interpretation; Contribution to manuscript preparation; Contribution to critical revision, adding intelectual content.

\section{Conflicts of interest}

The authors declare that they have no conflict of interest related to the publication of this manuscript.

\section{References}

BAZANELLA, S.I. 2003. Diversidade de Bacillariophyceae em rios encachoeirados do Parna, Chapada dos Guimarães - Mato Grosso. Trabalho de conclusão de curso, Universidade Federal de Mato Grosso, Cuiabá.

BELLINGER, E.G. \& SIGEE, D.C. 2010. Freshwater algae: identification and use as Bioindicators. Edição Wiley-Blackwell, West Sussex. 285p. ISBN 978-0-470-05814-5
BICUDO, C.E.M \& MENEZES, M.A. 2006. Gêneros de algas continentais do Brasil: chave para identificação e descrições. 2 ed. Rima, São Carlos.

BICUDO, C.E.M. \& MENEZES, M. 2010. Introdução: as algas do Brasil. In Catálogo de plantas e fungos do Brasil (R.C. Forzza et al., eds). Andrea Jakobsson Estúdio/Instituto de Pesquisa Jardim Botânico do Rio de Janeiro, Rio de Janeiro, v.1, p.49-60.

BITTENCOURT-OLIVEIRA, M.C., PICCIN-SANTOS, V., MOURA, A.N., ARAGÃO-TAVARES, N.K.C. \& CORDEIRO-ARAÚJO, M.K. 2014. Cyanobacteria, microcystins and cylindrospermopsin in public drinking supply reservoirs of Brazil. Ann. Acad. Bras. Cienc. 86(1):297-310. doi:10.1590/0001-3765201302512

BORGES, O. 1903. Die algen der ersten Regnellschen Expedition: II.: Desmidiaceen. Arkiv För Botanik, n. 1: p. 277-285.

BRASIL. Portaria $\mathrm{n}^{\circ} 1469$, de 29 de Dezembro de 2000. Estabelece os procedimentos e responsabilidades relativos ao controle e vigilância da qualidade da água para consumo humano e seu padrão de potabilidade. Diário Oficial [da] República Federativa do Brasil, Brasília, DF, 22.02.01, Seção I.

CAVALCANTE, K.P., TREMARIN, P.I., CASTRO, E.C., TIBIRICÁ, C.E.J.A., WOJCIECHOWSKI, J., LUDWIG, T.A.V. Epiphytic Eunotia (Bacillariophyceae) on Podostemum from Santa Catarina, southern Brazil, including new observations on morphology and taxonomy of some rare recorded species. Biota Neotropica. 14(3): 000-000. http://dx.doi. org/10.1590/1676-06032014003 (last access in 02/03/2018)

COSTA, R.L., TODESCHINI, T., RIBEIRO, M.J.P. \& TEIXEIRA-OLIVEIRA, M. 2017. Florações de cianobactérias potencialmente tóxicas em tanques de pisciculturas da região centro sul do estado de mato grosso. Biodiversidade. 16 (1): 33-45.

DE-LAMONICA-FREIRE, E.M. 1989. Catálogo das algas referidas para o Estado de Mato Grosso, Brasil, 2. Rev. Brasil., Biol. 49 (3): 679-689.

DIAS, I.C.A. 1986. Zygnemaceae (Zygnemaphyceae) da Chapada dos Guimarães e arredores, Mato Grosso, Brasil: uma contribuição ao seu conhecimento. Rickia 13: 69-75.

FONSECA, L.W., ALVES, M.A.S., SILVA, L.C.M., RODRIGUES, N.S. \& PINILLOS, A.C.M. 2014. Zygnemaphyceae do Córrego Quinera - Parque Nacional de Chapada dos Guimarães/MT: estudo qualitativo e quantitativo. Enciclopédia Biosfera, Centro Científico Conhecer 10 (18): 3107-3117.

FREITAS, L.C \& LOVERDE-OLIVEIRA, S.M. 2013. Checklist of green algae (Chlorophyta) for the state of Mato Grosso, Central Brazil. Check List 9 (6): 1471-1483.

GOMES-SILVA, P.A.J. 2015. Limnologia e qualidade da água da bacia do rio Coxipó (MT): subsídios à gestão dos recursos hídricos. Dissertação de Mestrado, Universidade Federal de Mato Grosso, Cuiabá.

GUERRA, A.B., TONUCCI, M.C., CEBALLOS, B.S.O., GUIMARÃES, H.R.C., LOPES, W.S., AQUINO, S.F. \& LIBÂNIO, M. 2015. Remoção de microcistina-LR de águas eutrofizadas por clarificação e filtração seguidas de adsorção em carvão ativado granular. Eng Sanit Ambient. 20 (4): 603-612.

KOMÁREK, J. 2014. Modern classification of cyanobacteria. In Cyanobacteria: An Economic Perspective (eds N. K. Sharma, A. K. Rai and L. J. Stal), John Wiley \& Sons, Ltd, Chichester, UK. p. 21-39. doi: 10.1002/9781118402238. ch2

LIMA, E.B.N.R. \& LIMA, J.B. 2009. Qualidade da água das principais subbacias urbanas do município de Cuiabá. In Bacia do rio Cuiabá: uma abordagem socioambiental (D.M. Figueiredo \& F.X.T. Salomão, orgs.). Entrelinhas: EdUFMT, Cuiabá, p. 140-154

LOPES, L.E., PINHO, J.B., BERNARDON, B., OLIVEIRA, F.F., BERNARDON, G., FERREIRA, L.P., VASCONCELOS, M.P., NOBREGA, P.F.A. \& RUBIO, T.C. 2009. Aves da Chapada dos Guimarães, Mato Grosso, Brasil: uma síntese histórica do conhecimento. Papéis Avulsos de Zoologia, Museu de Zoologia da Universidade de São Paulo 49(2): 9-47.

MARCAL, S.F. \& LOVERDE-OLIVEIRA, S.M. 2015. Phytoplankton in Coqueiro lake (Pantanal de Poconé, Mato Grosso, Brazil). Revista Biotemas, 28 (2): 9-25. 
MENEZES, M. 1986. Ficoflórula da Chapada dos Guimarães e arredores, Mato Grosso, Brasil: Euglenaceae pigmentadas (Euglenophyceae). Rickia 13: 87-95.

MENEZES, M. \& FERNANDES, V. 1990. Dinophyceae do Estado de Mato Grosso, MT, Brasil: Municípios de Barra do Bugres, Cáceres, Chapada dos Guimarães, Porto Esperidião e Quatro Marcos. Acta bot. bras. 4 (2): 21-30.

MENEZES, V.C., BUENO, N.C., BORTOLINI, J.C., BIOLO, S. \& SIQUEIRA, N.S. 2011. O gênero Cosmarium Corda ex Ralfs (Desmidiaceae) no Reservatório de Itaipu, PR, Brasil. Hoehnea 38 (3): 483-493.

MENEZES, M. et al. 2015. Update of the Brazilian floristic list of Algae and Cyanobacteria. Rodriguésia. 66 (4): p. 1047-1062.

MORESCO, C. \& RODRIGUES, L. 2013. O perifiton como bioindicador em rios. In Ecologia do Perífiton (A. Schwarzbold, A.L. Burliga, L.C. Torgan, orgs.). Rima, São Carlos, v.1, p.147-156.

NISHIMURA, P.Y.; MOSCHINI-CARLOS, V., POMPÊO, M. 2015. O estudo fitoplâncton com base nos grupos funcionais: origens e um vislumbre sobre seu futuro. In Ecologia de reservatórios e interfaces (M. Pompêo, V. Moschini-Carlos, P. Yuri Nishimura, S. Cardoso da Silva, J. Cesar LópezDoval, orgs.). Instituto de Biociências da Universidade de São Paulo, São Paulo, p.120-131.

PADILHA JUNIOR, A.G \& NUNES, J.R.S. 2014. As Variações da Qualidade da Água na Bacia do Rio Coxipó, Cuiabá-MT. Uniciências, Cuiabá 18 (1): 57-66.
REYNOLDS, C. S. 2006. Ecology of phytoplankton. Cambrigde: Cambrigde University Press.

ROUND, F.E.; CRAWFORD, R.M. \& MANN, D.G. 1990. The diatoms: biology and morphology of genera. Cambridge, Cambridge University Press.

RUGGIERO, M.A.; GORDON, D.P.; ORRELL, T.M.; BAILLY, N.; BOURGOIN, T.; BRUSCA, R.C.; CAVALIER-SMITH, T.; GUIRY, M.D.; KIRK. P.M. 2015. A Higher Level Classification of All Living Organisms. PLoS ONE, 10(4): e0119248. 60 pp. doi:10.1371/journal.pone.0119248.

SOPHIA, M.G. \& SILVA, L.H.S. 1989. Considerações sobre a flora de Desmídias filamentosas (Zygnematophyceae) do Noroeste de Mato Grosso e Sudeste de Rondônia, Brasil. Revista Brasileira de Biologia 49 (4): 943-956.

SOUSA, S.F. 2004. Ocorrência de microalgas e cianobactérias em ambientes fitotélmicos bromelícolas do Parque Nacional de Chapada dos Guimarães - MT. Trabalho de Conclusão de Curso. Universidade Federal de Mato Grosso, Cuiabá.

STEVENSON, R.J. 1996. Na Introduction to algal ecology in freshwater benthic habitats. In Algal ecology: freshwater bentic ecosystems. (R.J, Stevenson, M.L, Bothwell, R.L, Lowe, eds) San Diego: Academic Press, cap.1, p.3-30.

TEIXEIRA DE OLIVEIRA, M. \& HARDOIM, E.L. 2010. Study of testacean assemblages (Protozoa: Rhizopoda) in touristic waterfall regions of Chapada dos Guimarães National Park, Mato Grosso State, Brazil. Acta Scientiarum. Biological Sciences. 32 (4): 387-395. 